\title{
Healthcare System
}

\author{
Mr Kunal Nabariya ${ }^{1}$, Miss Dnyanashree Patil ${ }^{2}$, Miss Prachi Solanki ${ }^{3}$ \\ Comp Dept, Stes, Sinhgad Academy of Engineering, Pune, India ${ }^{1,2,3}$
}

\begin{abstract}
As seen in the past few decades, it is very common to observe the patient's paper work at the hospital. Even though the same personal information is used, an unusual way to actually decrement the amount of these paper works does not exist. The development of mobile web provides development direction for medical industry and a new service mode. In this paper, we introduce e-health authentication system to obtain patient's health record easily and securely in the local hospital and also to reduce the redundant paper work. One of the aims of this project is to use the dataset and machine learning techniques to predict the type of disease based on the symptoms and patient's health report. A mail regarding login details which includes patient's mail-id, secret key and symptoms is sent to the doctor automatically via email. The doctor logs-in into the application where predicted disease and personal information of patient is obtained. Further the doctor sends prescription to the patient which is seen by the pharmacist with the help of mailed secret-key .Here, we describe an integrated system, developed for use by the healthcare personnel within healthcare facilities, adapted to all handheld devices. With our proposed scheme, we believe that it will improve efficiency in terms of the cost and time for the patient, hospital and the doctor and protect patient's personal information.
\end{abstract}

Keywords: Healthcare System, Disease Prediction, Doctor Recommendation, Machine Learning

\section{INTRODUCTION}

Medical data is an ever growing source of information generated from hospitals consisting of patient records in the form of hard copies which can be made easier and convenient by using this healthcare system application. Our aim is to build a Health-care Portal system which will provide the features like clinical management, patient records, disease prediction and doctor recommendation for every patient as per their updated disease information. Disease is being predicted by one of most accurate ML classification algorithms such as Ada-boost classifier, K-Nearest neighbour, Random-forest, Gradient Boost, sLogistic Regression, Support Vector Classifier and doctor recommendation by Haversine algorithm. Keeping track of all the activities and records is very error prone for the hospital authorities. It is also very inefficient and time consuming process. Recording and maintaining the records are highly unreliable. It is also not economically and technically feasible to maintain the records on paper. The main aim of project is to provide paperless up to $90 \%$. It also aims at providing low cost and reliable automation of the existing system. The patient registers himself in the system and enters all his personal details along with symptoms and attaches reports if any. A mail regarding login details which includes patient's mail-id, secret key and symptoms is sent to the doctor automatically via email. The doctor logs-in into the application where predicted disease and personal information of patient is obtained. Thus, the doctor sends prescription to the patient which is seen by the pharmacist with the help of mailed secret-key.

\section{LITERATURE SURVEY}

\section{Design and Implementation of Doctor-Patient Interaction System Based on Android}

Authors: Ran Wei, Zhimin Yang

Highlights: It is presentation of doctor-patient interaction system based on Android. Its glorious performance on mobile terminals makes it potential that patients area unit able to access the hospital server to get the mandatory suggestion regarding the symptoms and move with the doctors on their own mobile terminals, whereas doctors will track patients whenever and where potential or build a diagnosing of alert depends on the observation knowledge from the hardware of mobile terminals.

Limitations: System has shortcomings, such as in the monitoring module, when the objects in the camera changes in a large scope, the amount of coded data increases quickly which will cause the system efficiency decreases.

\section{Secure Transmission Medical Data for Pervasive Healthcare System using Android}

Authors: Sudha.G and Ganesan.R

Highlights: It is created for accessing the medical multimedia data of patients when the user is in mobility. The mobile have lesser memory to store a data. So to access the larger database with security, we use the My Sql database in server 
Vol. 8, Issue 5, May 2019

system. This connectivity is established with the help of server program. It ensures authentication and security in accessing database.

Limitations: The mobile application can be developed with context aware, adaptability, delay should be decreased.

3. Planning and Development of an Electronic Health Record Client based on the Android Platform

Authors: Dimitris Tychalas, Athanasios Kakarountas

Highlights: The aim of this work is to present the system's client, which operates on a mobile device Android based and acquires data from the eHR connecting to a centralized Database offered by the Hospital or Clinic.

Limitations: Network manager is needed, if any part of network fails a lot of disruption occurs.

\section{FASE: Fast Authentication System for E-health}

Authors: Mungyu Bae, Suk Kyu Lee, Seungho Yoo, and Hwangnam Kim

Highlights: Proposed QR code authentication scheme for e-health, with this proposed scheme, patient can reduce the redundant process of writing paper for visiting hospital for diagnosis and a local hospital can manage patient's personal information cost-effectively.

Limitations: Disease is not predicted ,so every time patient has to inform doctor about his/her symptoms.

III.

PROPOSED SYSTEM

We have proposed system in such a way that a healthcare system is developed wherein disease prediction, doctor recommendation and medicine prescription is done .It is an application in which - There are three modules namely Patient, Doctor and Medical. In patient module, patient registers himself by entering all his personal details. He can then attach reports, enter symptoms and disease is predicted by the most accurate machine learning algorithm. Further, he can search for doctor either by his disease type or any doctor in his/her vicinity. Symptoms and login-details are mailed to doctor. In doctor's module, doctor registers himself and logs-in into the application where predicted disease and personal information of patient is obtained. Further the doctor sends prescription to the patient. In medical module, pharmacist logs-in and then with the help of mailed secret-key sees the prescription and gives medicines.

\section{SYSTEM ARCHITECTURE}

Following diagram is our system's architecture diagram:

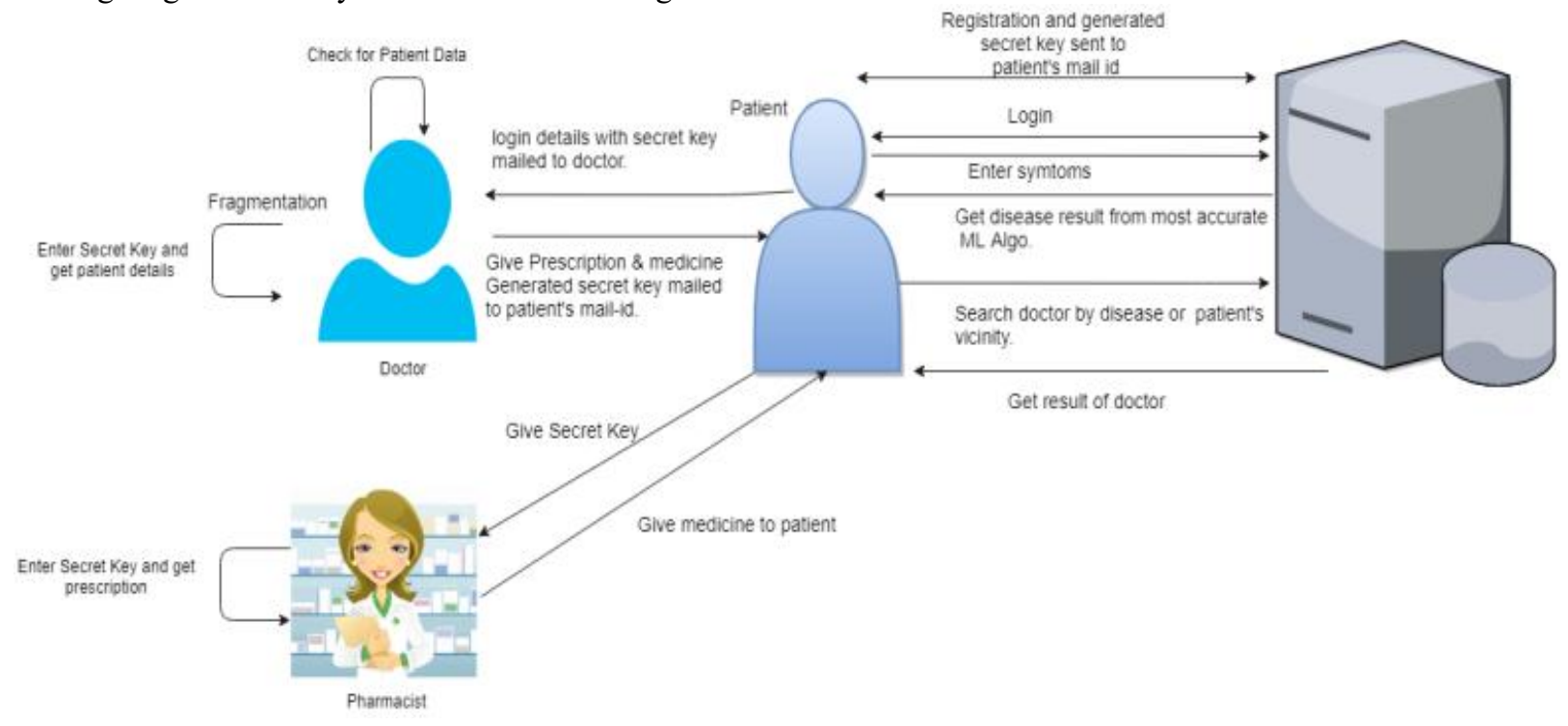

In our System architecture following steps are performed:

1. Patient registers himself by entering personal details .

2. Patient enters symptoms and disease is predicted by most accurate machine learning algorithm.

3. Patient searches for doctor by disease or doctor in his/her vicinity and mail is sent to doctor including login details with secret key. 
Vol. 8, Issue 5, May 2019

4. Doctor logs-in into the system and accept pending patient requests and get patient details by entering secret key.

5. Doctor prescribes medicine and mails back to patient.

6. Pharmacist enters secret key and gets prescription and gives medicines to patient.

\section{METHODOLOGIES}

\subsection{Dataset:}

Dataset we have used is a public dataset. The dataset initially contained 2 features: disease and its symptoms. It was not up-to our requirement, so main aim was converting unstructured data to structured format. We did it by encoding it into binary format and pre-processing of data was done. Training and testing data has been randomly split. After application of various machine learning classification algorithms, the most accurate algorithm is predicting the disease. Unstructured dataset and encoded structured dataset is shown in images below.

\begin{tabular}{|c|c|c|}
\hline 4 & A & B \\
\hline 1 & Disease & Symptom \\
\hline 2 & UMLS:C0020538_hypertensive disease & UMLS:C0008031_pain chest \\
\hline 3 & & UMLS:C0392680_shortness of breath \\
\hline 4 & & UMLS:C0012833_dizziness \\
\hline 5 & & UMLS:C0004093_asthenia \\
\hline 6 & & UMLS:C0085639_fall \\
\hline 7 & & UMLS:C0039070_syncope \\
\hline 8 & & UMLS:C0042571_vertigo \\
\hline 9 & & 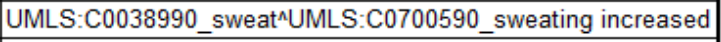 \\
\hline 10 & & UMLS:C0030252_palpitation \\
\hline 11 & & UMLS:C0027497_nausea \\
\hline 12 & & UMLS:C0002962_angina pectoris \\
\hline 13 & & UMLS:C0438716_pressure chest \\
\hline 14 & UMLS:C0011847_diabetes & UMLS:C0032617_polyuria \\
\hline 15 & & UMLS:C0085602_polydypsia \\
\hline 16 & & UMLS:C0392680_shortness of breath \\
\hline 17 & & UMLS:C0008031_pain chest \\
\hline 18 & & UMLS:C0004093_asthenia \\
\hline 19 & & UMLS:C0027497_nausea \\
\hline 20 & & UMLS:C0085619_orthopnea \\
\hline 21 & & UMLS:C0034642_rale \\
\hline 22 & & 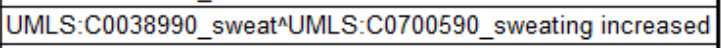 \\
\hline 23 & & UMLS:C0241526_unresponsiveness \\
\hline 24 & & UMLS:C0856054_mental status changes \\
\hline 25 & & UMLS:C0042571_vertigo \\
\hline
\end{tabular}

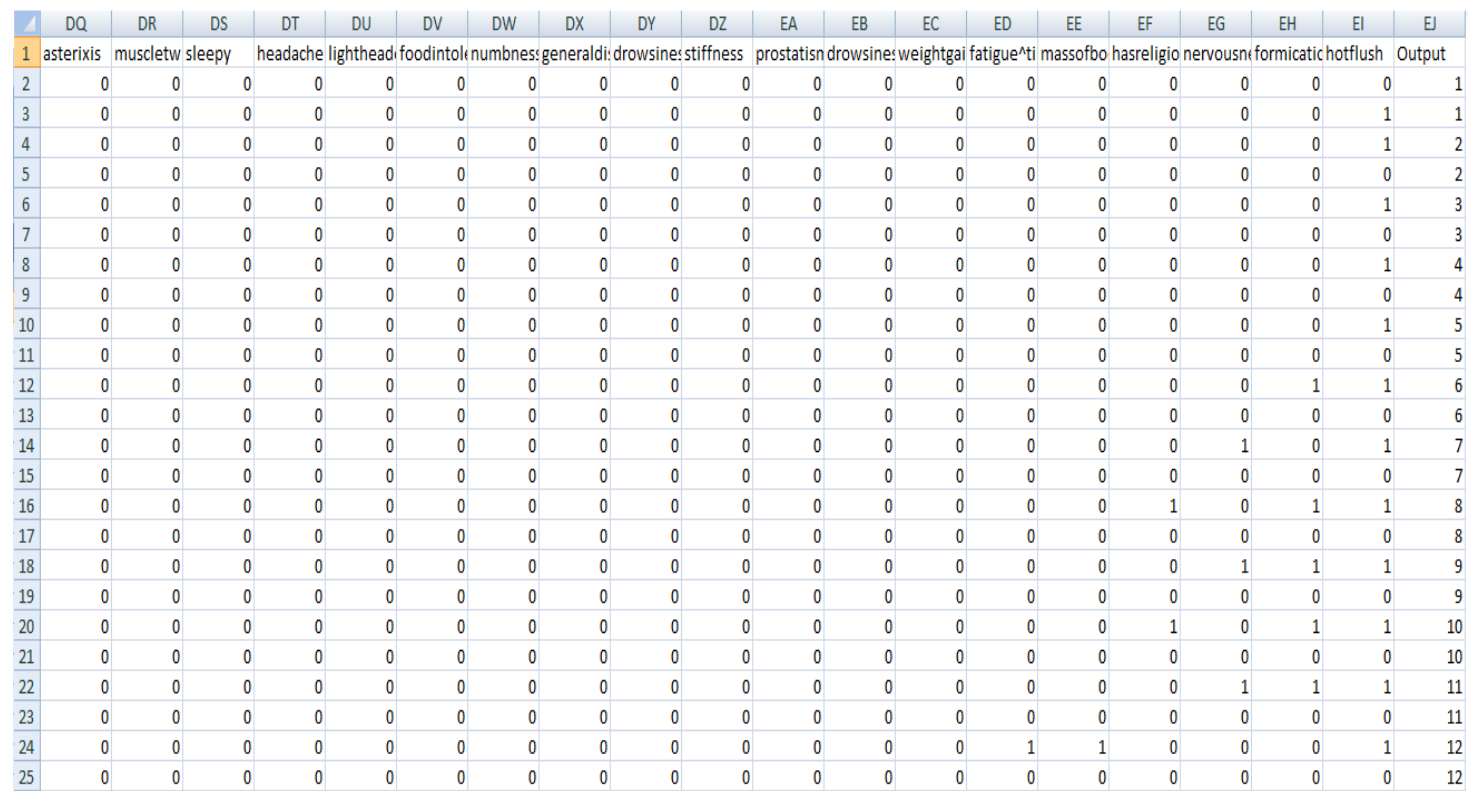


Vol. 8, Issue 5, May 2019

\subsection{Algorithms:}

We have implemented different Machine learning classification algorithms on our dataset .Algorithms such as Adaboost classifier ,K-Nearest neighbour, Random-forest, Gradient Boost ,Logistic Regression, Support Vector Classifier have been used. Most accurate algorithm's output is considered and respective disease is being predicted accordingly.

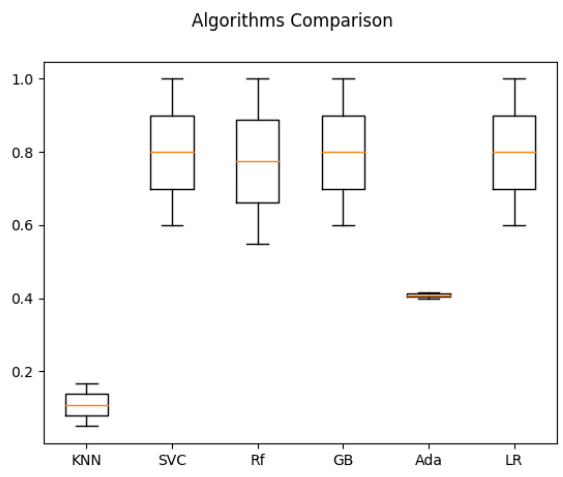

VI. ADVANTAGES

- $\quad$ The medical process will be easy and most secure.

- The process of fake prescription will be overcome.

- $\quad$ Doctors can cure patients from long distance.

- $\quad$ No need to carry the whole documents in a single file

\section{DISADVANTAGES}

- Internet connectivity is weak in village areas.

- $\quad$ All patients should be aware of internet

\section{CONCLUSION}

We have proposed health care system for patient. For the same, we have used different Machine learning classification algorithms for disease prediction as well as Haversine algorithm for recommending nearby doctors. Also, specialized doctors are recommended in order to maintain distant communication between doctor and the patient. Our protocols utilize simple technologies available in most out-of-the box Smartphone devices. In addition, we will study methods for improving the security and user experience by means of visualization in other contexts. With our proposed scheme, patient can reduce the redundant process of writing paper for visiting hospital for diagnosis and doctors can manage patient's personal information cost-effectively. We believe that our proposed method can be utilized as a secure communication medium for the patient and the e-health system.

\section{RESULT}

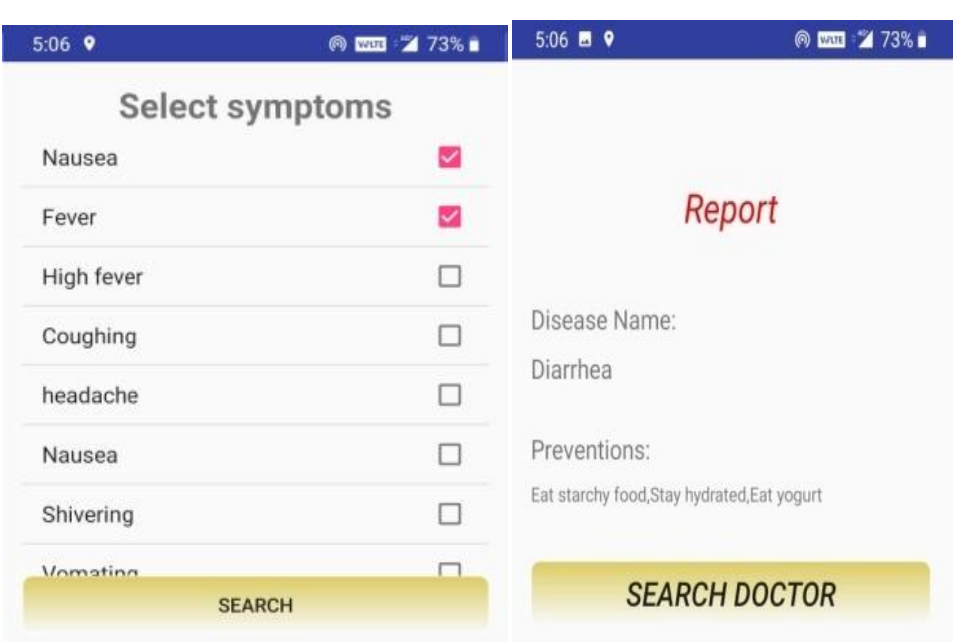


Vol. 8, Issue 5, May 2019

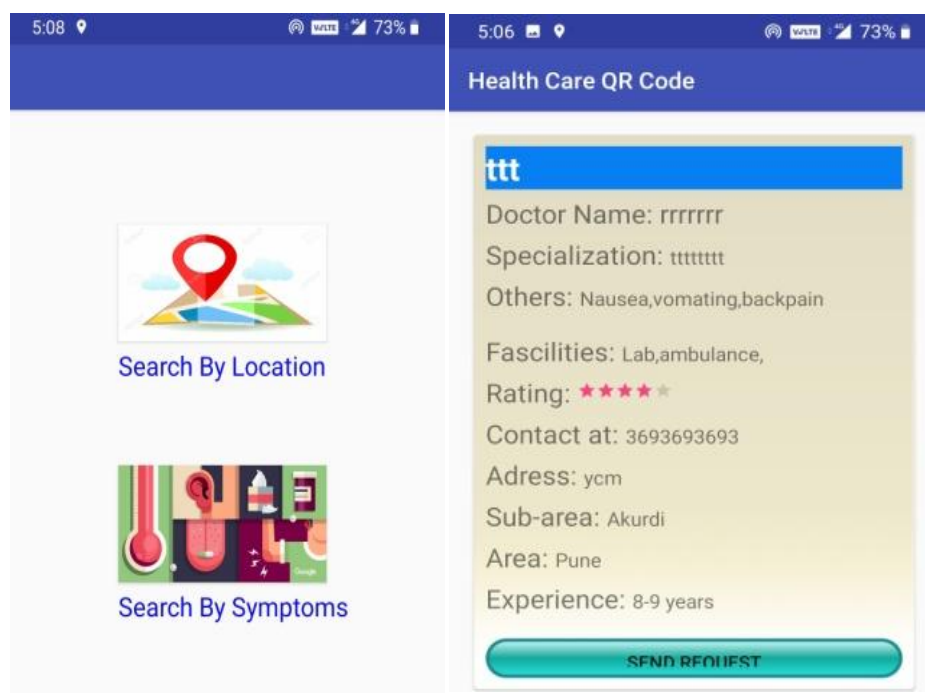

\section{REFERRENCES}

[1]. 2013 IEEE 17th International Symposium on Consumer Electronics (ISCE) "Design of Mobile Healthcare Service with Health Records Format Evaluation "Hung-Ming Chen*, Yong-Zan Liou, Shih-Ying Chen, Jhuo-Syun Li

[2]. 2012 INTERNATIONAL SYMPOSIUM ON INFORMATION TECHNOLOGY IN MEDICINE AND EDUCATION "Design and Implementation of Doctor-Patient Interaction System Based on Android " Ran Wei, Zhimin Yang

[3]. 2016 15th IEEE International Conference on Machine Learning and Applications "A Big Data Analytics Framework for Supporting Multidimensional Mining over Big Healthcare Data" ,Mario Bochicchio Salento Lecce, Alfredo Cuzzocrea DIA .

[4]. Proceedings of the International Conference on Intelligent Sustainable Systems (ICISS 2017) IEEE Xplore "Analysis and Prediction of Breast cancer and Diabetes disease datasets using Data mining classification Techniques" Deepika Verma ,Dr. Nidhi Mishra (Author).

[5]. $20144^{\text {th }}$ International Conference on Computer and Knowledge Engineering” Disease Detection in Medical Prescriptions Using Data Mining Tools" Mahsa Soudi Alamdari, Mehdi Teimouri, Amir Hashemi-Meshkini

[6]. "Data mining for better healthcare: A path towards automated data analysis?”, Tania Cerquitelli*, Elena Baralis*, Lia Morra $\uparrow$ and Silvia Chiusano ,Control and Computer Engineering Dept., Politecnico di Torino, ITALY

[7]. 2015 IEEE/ACM International Conference on Advances in Social Networks Analysis and Mining " Importance of Data Mining in Healthcare: A Survey" Mohammad Hossein Tekieh1, Bijan Raahemi1

[8]. 2012 International Conference on Computer Science and Service System , "The Use of Data Mining in Pharmic Quality Analysis of Traditional Chinese Medicines* " Xia WU1 Shun LONG, Wei-heng ZHU "

[9]. IEEE ICC 2013 - Selected Areas in Communications Symposium "NFC Based m-Healthcare Application Focusing on Security, Privacy and Performance" Weider D. Yu, Hargun Hansrao , Kirandeep Dhillon, Pradeep Desinguraj 